\title{
Hard physics in STAR
}

\section{Thomas Dietel ${ }^{* \dagger}$}

Johann-Wolfgang-Goethe Universität Frankfurt am Main, Germany

E-mail: tomedietel.net

The hot and dense matter created in high-energy nuclear collisions is believed to undergo a transition into a deconfined phase where partonic degrees of freedom determine the dynamics of the medium. High- $p_{\perp}$ partons, that are produced in the initial collisions between nucleons of the incoming nuclei, lose energy as they propagate through the medium. This effect, called jetquenching, is observed in high- $p_{\perp}$ particle spectra, in azimuthal correlations with the reaction plane (elliptic flow) and jet-like two-particle correlations.

STAR consists of tracking detectors and electromagnetic calorimetry with large and azimuthally symmetric acceptance and is exceptionally well suited for single particle detection and correlation studies at high $p_{\perp}$. In the last five years, it has collected a large dataset including $\mathrm{Au}+\mathrm{Au}$ and $\mathrm{Cu}+\mathrm{Cu}$ collisions at different energies and reference data from $\mathrm{p}+\mathrm{p}$ and $\mathrm{d}+\mathrm{Au}$ collisions.

We present particle spectra and two-particle correlations at high- $p_{\perp}$, and relate these measurements to the properties of the medium.

International Europhysics Conference on High Energy Physics

July 21st - 27th 2005

Lisboa, Portugal

\footnotetext{
*Speaker.

${ }^{\dagger}$ For the STAR Collaboration (http: //www. star.bnl.gov/).
} 


\section{Introduction}

Collisions of heavy ions at high energies produce a hot and dense system of strongly interacting particles. At high energy densities, a new phase of deconfined matter is expected, the $\mathrm{QGP}^{1}$. RHIC $^{2}$ data show evidence for this phase transition in $\mathrm{Au}+\mathrm{Au}$ collisions at $\sqrt{s_{N N}}=200 \mathrm{GeV}$ [1]. This article shows new results in hard physics at $\mathrm{STAR}^{3}$ since the publication of an extensive review of the first three years of RHIC running by STAR [1]. Soft particle production will be covered by another article in these proceedings [2].

Hard partons with high transverse momentum are produced in the very first collisions between partons of the incoming nuclei, and their initial production is not affected by the hot medium. The interactions of the energetic partons with the color charges in the medium lead to a large energy loss of the traversing partons and a strong suppression of hard particles from the fragmentation. This suppression has been observed by STAR [3, 4] and was demonstrated not to be due to initial state effects [5]. Partonic energy loss can also explain the disappearance of azimuthal back-to-back correlations of high- $p_{\perp}$ particles: dijets that are the source of these correlations are suppressed in central $\mathrm{Au}+\mathrm{Au}$ collisions due to energy loss of the away-side jet [6].

\section{Au+Au Collisions at $\sqrt{s_{N N}}=62 \mathrm{GeV}$}

The presence of strong high- $p_{\perp}$ suppression in $200 \mathrm{GeV} \mathrm{Au+Au} \mathrm{collisions} \mathrm{immediately} \mathrm{opens} \mathrm{the}$ question about the minimum collision energy and system size required for this effect. RHIC features a program to scan for this onset, including a run at a reduced energy of $\sqrt{s_{N N}}=62 \mathrm{GeV}$. This section will summarize the high- $p_{\perp}$ measurements from the this run.

Figure 1 shows the nuclear modification factor $R_{A A}$ in $\mathrm{Au}+\mathrm{Au}$ collisions at $\sqrt{s_{N N}}=62$ and $200 \mathrm{GeV}$. The suppression in $62 \mathrm{GeV}$ collisions shows similar patterns as in $200 \mathrm{GeV}$ collisions: a monotonic increase up to $p_{\perp} \approx 2 \mathrm{GeV} / c$, a saturation in peripheral collisions, and a decrease in central collisions, down to

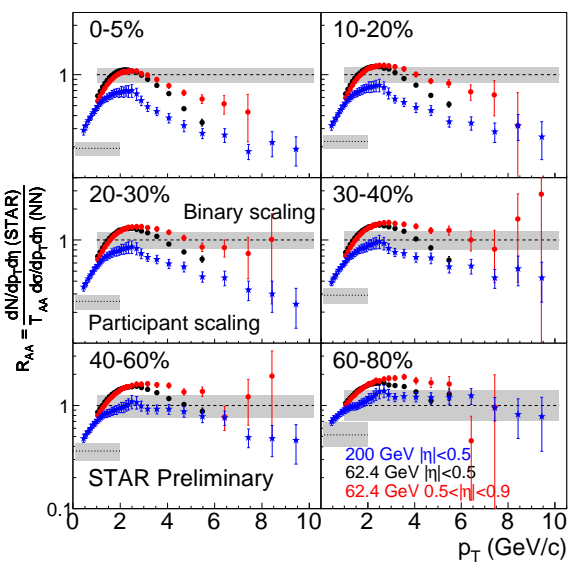

Figure 1: Nuclear modification factor $R_{A A}$ for $\mathrm{Au}+\mathrm{Au}$ collisions at $\sqrt{s_{N N}}=62$ and $200 \mathrm{GeV}$ [7]. a suppression by almost a factor of 5 for particles with $p_{\perp}>6 \mathrm{GeV} / c$. The suppression is slightly smaller in $62 \mathrm{GeV}$ collisions, indicating a smaller energy loss in the medium at the lower energy.

Azimuthal correlations from $62 \mathrm{GeV}$ collisions are shown in figure 2. A trigger particle is chosen with $4<p_{\perp}<6 \mathrm{GeV} / c$, and correlated with particles with $p_{\perp}>2 \mathrm{GeV} / c$. The distribution of the azimuthal angle between trigger and associated particles shows a peak around $\Delta \phi \approx 0$ from particles from the same jet, and a second peak at $\Delta \phi \approx \pi$ due to back-to-back dijets. The difference of the near-side yield per trigger particle in 62 and $200 \mathrm{GeV}$ collisions is due to different kinematic

\footnotetext{
${ }^{1}$ Quark-Gluon-Plasma

${ }^{2}$ Relativistic Heavy Ion Collider

${ }^{3}$ Solenoidal Tracker At RHIC
} 
conditions in the jet production and fragmentation [8], but does not change as a function of centrality. The away-side peak visible in peripheral collisions is strongly suppressed in central collisions, without statistically significant differences between low- and high-energy collisions.

The data from the $62 \mathrm{GeV}$ run is qualitatively similar to the full-energy data, but shows smaller high- $p_{\perp}$ suppression. This indicates, that the same physical processes apply, and that different energy densities of the produced medium lead to a smaller energy loss.

\section{3. $\Delta \eta$ Correlations}

Jets result not only in azimuthal correlations, but also in correlations in pseudo-rapidity $\eta$. As there is no direct correlation between the pseudo-rapidities of two opposite jets, the analysis of these correlations is restricted to modifications of the near-side jet.

Figure 3 shows the dependence of the width of the near-side peak in correlations, determined by a fit to the background-subtracted $\Delta \eta$ distribution. $\mathrm{p}+\mathrm{p}$ and $\mathrm{d}+\mathrm{Au}$ collisions agree well, but $\mathrm{Au}+\mathrm{Au}$ collisions show a significant broadening of the peak. The effect decreases with $p_{\perp}$ of the trigger particle, and disappears for the highest bin. The associated yield per trigger particle is not affected by this broadening and is the same for all systems. The broadening is not present in azimuthal correlations, indicating an anisotropy of the hot medium created in the collision. It has been suggested that this anisotropy is due to the flow of the medium along the beam direction [9]. For final conclusions, detailed comparisons between model calculations and a higher statistics data set are needed.

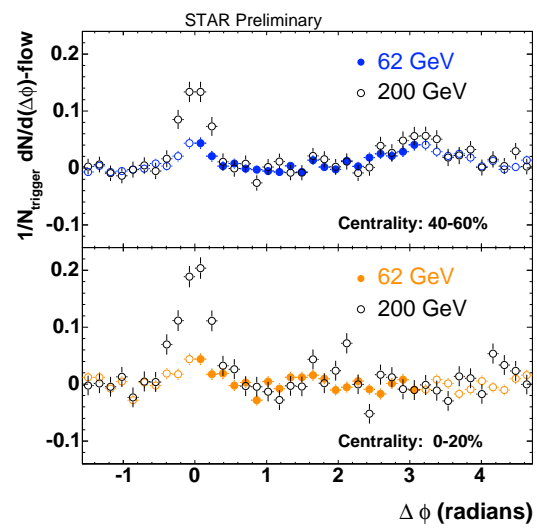

Figure 2: Azimuthal correlations in $\mathrm{Au}+\mathrm{Au}$ collisions at 62 and $200 \mathrm{GeV}$ for $4<p_{\perp}$ (trigger $)<6 \mathrm{GeV} / c$ and $2<$ $p_{\perp}($ assoc $)<4 \mathrm{GeV} / c$ [7].

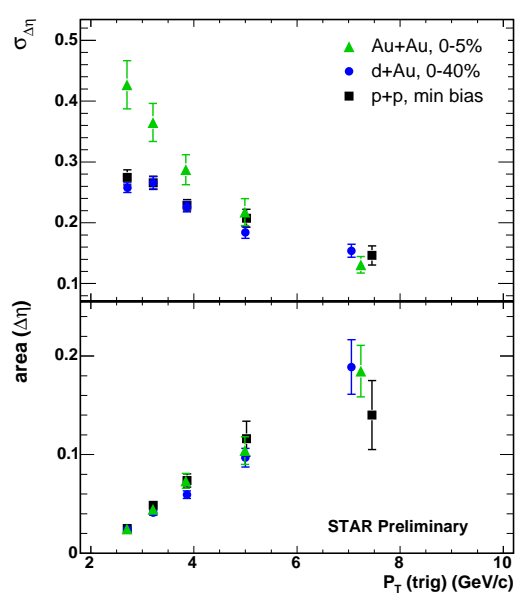

Figure 3: Width and yield of nearside $\Delta \eta$ correlation peak as function of $p_{\perp}$ (trigger), for $2 \mathrm{GeV} / c<$ $p_{\perp}($ assoc $)<p_{\perp}($ trigger $)$.

\section{Azimuthal Correlations with low- $p_{\perp}$ associated Particles}

Since energy and momentum of the fragmenting parton have to be conserved, the "lost" energy cannot disappear, but has to reemerge in softer particle production. This effect was not visible in previous correlation analyses, that were restricted to associated particles with high transverse momentum. By lowering the transverse momentum of the associated particles, correlation analyses can become sensitive to these modifications [10].

Figure 4 shows the modification of $\Delta \eta$ - and $\Delta \phi$-correlations between hard trigger and soft associated particles. The broadening of the near-side $\Delta \eta$-peak is visible in the right panels and 
even more pronounced for associated low- $p_{\perp}$ particles. The larger integral under the near-side $\Delta \eta$ and $\Delta \phi$-peaks indicates an increased yield of associated particles per trigger particle due to energy loss of the hard parton.

In away-side $\Delta \phi$-correlations, the disappearance at high- $p_{\perp}$ is contrasted by an increase and broadening at low$p_{\perp}$ : particle production is shifted from high to low transverse momentum. The peak is broadened and shows a deviation from the usual Gaussian shape, that has been speculated to be due to shock waves in the medium [11].

\section{Summary}

Since the establishment of partonic energy loss as the cause of high- $p_{\perp}$ particle suppression, as described in [1], high- $p_{\perp}$ physics is attempting to pro-

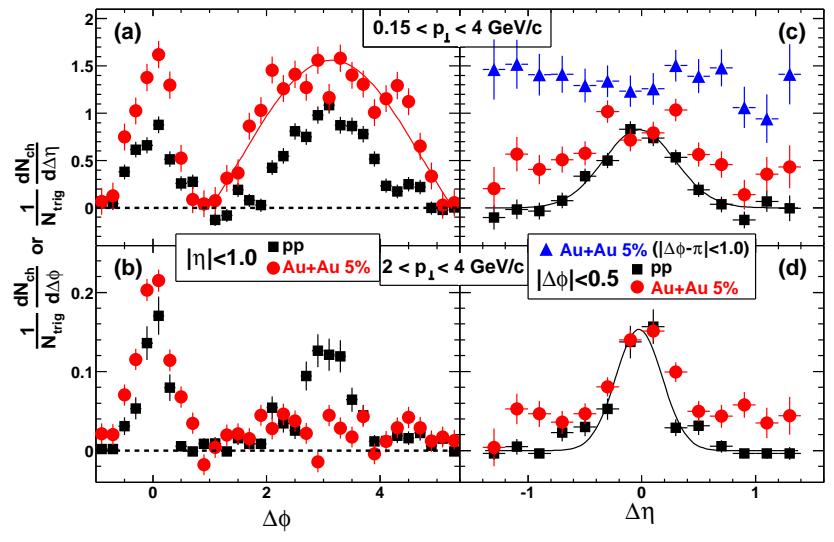

Figure 4: $\Delta \phi$ (left) and $\Delta \eta$ (right) correlations in $\mathrm{p}+\mathrm{p}$ and $\mathrm{Au}+\mathrm{Au}$ collisions at $200 \mathrm{GeV}$ for all associated particles $\left(0.15<p_{\perp}<4 \mathrm{GeV} / c\right.$, top) and associated high- $p_{\perp}$ particles $\left(2<p_{\perp}<4 \mathrm{GeV} / c\right.$, bottom). Trigger particles have $4<p_{\perp}<6 \mathrm{GeV} / c$. [10]

vide a better and more quantitative understanding of the interaction of a parton with the medium. Collisions with different ion species and energies will provide information about the dependence of the energy loss on the energy density and path-length in the medium. The modification of jet properties like the broadening of $\Delta \eta$ correlations and the strong modification of correlations between hard trigger and soft associated particles can provide further constraints for theoretical models of partonic energy loss. Data from a high-statistics RHIC run at $\sqrt{s_{N N}}=200 \mathrm{GeV}$, which is becoming available now, will allow to extend the study of hard physics to even higher energies.

\section{References}

[1] J. Adams et al. Nucl. Phys., A757:102-183, 2005.

[2] L. Barnby. these proceedings.

[3] C. Adler et al. Phys. Rev. Lett., 89:202301, 2002.

[4] J. Adams et al. Phys. Rev. Lett., 91:172302, 2003.

[5] J. Adams et al. Phys. Rev. Lett., 91:072304, 2003.

[6] C. Adler et al. Phys. Rev. Lett., 90:082302, 2003.

[7] C. A. Gagliardi et al. Eur. Phys. J., C43:263-270, 2005.

[8] K. Filimonov, J. Phys. G 31, S513 (2005).

[9] N. Armesto, C. Salgado, and U. Wiedemann. Phys. Rev. Lett., 93:242301, 2004.

[10] J. Adams et al. Phys. Rev. Lett., 25:152301, 2005.

[11] J. Casalderrey-Solana, E. V. Shuryak, and D. Teaney. hep-ph/0411315 